\title{
Risk of stroke among patients with post-traumatic stress disorder: nationwide longitudinal study
}

Mu-Hong Chen, * Tai-Long Pan, * Cheng-Ta Li, Wei-Chen Lin, Ying-Sheue Chen, Ying-Chiao Lee, Shih-Jen Tsai, Ju-Wei Hsu, Kai-Lin Huang, Chia-Fen Tsai, Wen-Han Chang, Tzeng-Ji Chen, Tung-Ping Su and Ya-Mei Bai

\section{Background}

Previous evidence has shown positive associations between post-traumatic stress disorder (PTSD) and hypertension, dyslipidaemia and diabetes mellitus, which are all risk factors for stroke, but the role of PTSD in the subsequent development of stroke is still unknown.

\section{Aims}

To investigate the temporal association between PTSD and the development of stroke.

\section{Method}

Identified from the Taiwan National Health Insurance Research Database, 5217 individuals aged $\geqslant 18$ years, with PTSD but with no history of stroke, and 20868 age- and gender-matched controls were enrolled between 2002 and 2009, and followed up until the end of 2011 to identify the development of stroke.

\section{Results}

Individuals with PTSD had an increased risk of developing any stroke (hazard ratio (HR) 3.37, 95\% Cl 2.44-4.67) and ischaemic stroke ( $\mathrm{HR}=3.47,95 \% \mathrm{Cl} 2.23-5.39)$ after adjusting for demographic data and medical comorbidities. Sensitivity tests showed consistent findings (any stroke $\mathrm{HR}=3.02$, 95\% $\mathrm{Cl} 2.13-4.28$; ischaemic stroke $\mathrm{HR}=2.89,95 \% \mathrm{Cl} 1.79-4.66$ ) after excluding the first year of observation.

\section{Conclusions}

Individuals with PTSD have an increased risk of developing any stroke and ischaemic stroke. Further studies are required to investigate the underlying mechanisms.

\section{Declaration of interest}

None.
Post-traumatic stress disorder (PTSD) is a severe, disabling mental disorder that occurs in individuals who are exposed to traumatic events such as combat, severe physical or sexual assault, a serious accident, torture, sudden unexpected death of a loved one or natural disasters. ${ }^{1,2}$ The prevalence of PTSD in the USA is in the range $5-6 \%$ in men and $10-14 \%$ in women throughout their life, making it the fourth most common psychiatric disorder. ${ }^{2,3}$ In Taiwan a 3-year follow-up study surveying residents who had experienced a severe earthquake reported that the prevalence of PTSD was $8.3 \%$ at 6 months, $6.9 \%$ at 2 years and $6.5 \%$ at 3 years after the earthquake. ${ }^{4}$ Those with PTSD are at increased risk of suicide, psychiatric comorbidity and inability to work. ${ }^{1-4}$ In addition to psychiatric comorbidity, previous evidence has shown the negative impact of PTSD on some physical illnesses, and that individuals with PTSD had a higher likelihood of hypertension, dyslipidaemia and diabetes mellitus. ${ }^{5-8}$ Cohen et al assessed cardiovascular risk factors among US military veterans of the Iraq and Afghanistan wars and found an increased risk of hypertension, dyslipidaemia and diabetes mellitus among those with PTSD compared with those with no psychiatric disease. ${ }^{7}$ A population-based study in southern Germany demonstrated that individuals with PTSD were significantly associated with diabetes mellitus compared with those who had not experienced a traumatic event. ${ }^{9}$ Hypertension, dyslipidaemia and diabetes mellitus are all risk factors for stroke; however, studies investigating the role of PTSD and the subsequent development of stroke are limited and report inconsistent findings. ${ }^{10-13} \mathrm{~A}$ cross-sectional community-based study composed of 62 individuals with PTSD, 1669 with a trauma history but without PTSD and 1440 without a trauma history found that individuals with a trauma history and PTSD had a higher risk of stroke (odds ratio 1.2, 95\% CI $1.0-1.5) .{ }^{13}$ In contrast, Page \& Brass failed to validate this

*These authors contributed equally to the work. association $(\mathrm{OR}=1.13,95 \% \mathrm{CI}$ 0.66-1.91) among former World War II prisoners of war. ${ }^{12}$ The contradictory results in these two studies might be due to the small sample size, the cross-sectional study design and an ambiguous definition of PTSD diagnosis.

In this study we used a large sample size drawn from the Taiwan National Health Insurance Research Database and a longitudinal follow-up study design to investigate the temporal association between PTSD and stroke. We predicted that PTSD would increase the risk of developing stroke in later life.

\section{Method}

Taiwan's National Health Insurance, a mandatory universal health insurance programme, was implemented in 1995 and offers comprehensive medical care coverage to all Taiwanese residents. The National Health Research Institute (NHRI) is in charge of the entire insurance claims database, namely the National Health Insurance Research Database (NHIRD), which consists of healthcare data from more than $97 \%$ of the entire Taiwanese population (http://www.nhi.gov.tw/). The NHRI audits and releases the NHIRD for scientific and study purposes. Individual medical records included in the NHIRD are anonymous to protect patient privacy. Comprehensive information on insured individuals is included in the database, including demographic data, dates of clinical visits and disease diagnoses. The diagnostic codes used were based on the International Classification of Diseases, Ninth Revision, Clinical Modification (ICD-9-CM). ${ }^{14}$ The NHIRD has been used extensively in many epidemiological studies in Taiwan. $^{15-19}$

\section{Inclusion criteria}

Individuals aged 18 years or over who were identified as having newly diagnosed PTSD (ICD-9-CM codes 309.81) by 
board-certificated psychiatrists between 1 January 2002 and 31 December 2009, and who had no history of any stroke (ICD-9-CM codes 430-438) before enrolment, were included as the PTSD cohort. The time of PTSD diagnosis was defined as the time of enrolment. The age- and gender-matched (1:4) control cohort was randomly identified from among 1000000 individuals, after eliminating people who had been given a diagnosis of PTSD at any time and those with any stroke before enrolment. Diagnoses of any stroke (ICD-9-CM codes 430-438), ischaemic stroke (ICD-9-CM codes 433-435) and haemorrhagic stroke (ICD-9-CM codes 430-432) given by neurologists, neurosurgeons and emergency room doctors after the brain image examinations (computed tomography or magnetic resonance imaging) were identified during the follow-up (from enrolment to 31 December 2011 or death). Stroke-related medical comorbidities at enrolment and during the whole follow-up period (from enrolment to stroke onset or study end), including major depression, hypertension, dyslipidaemia, diabetes mellitus, renal disease, ischaemic heart disease, arrhythmia and head injury, were also identified in our study. In order to achieve diagnostic validity, the diagnoses of dyslipidaemia, diabetes mellitus, ischaemic heart disease and renal disease were given at least twice by corresponding physicians after laboratory examinations; the diagnosis of arrhythmia was given at least twice by corresponding physicians after examination of electrocardiograms; and the diagnosis of head injury was given at least twice by corresponding physicians after image examinations. However, major depression and hypertension were clinical diagnoses. So, patients with major depression diagnosed at least twice by psychiatrists and hypertension diagnosed at least twice by internal medicine doctors or family practitioners were included in our study. Level of urbanisation - from level 1 (most urbanised) to level 5 (least urbanised) - was also assessed for our study. ${ }^{20}$

\section{Statistical analysis}

For between-group comparisons the independent $t$-test was used for continuous variables and Pearson's chi-squared test for nominal variables, as appropriate. The Cox regression model was used to investigate the hazard ratio (HR) with $95 \%$ confidence interval of any stroke, ischaemic stroke and haemorrhagic stroke after adjusting for demographic data and medical comorbidities (at enrolment and during the whole follow-up) among patients with PTSD and the control group. Furthermore, we clarified whether the association between PTSD and the risk of stroke was consistent after we excluded patients who developed stroke shortly after the diagnosis of PTSD. Sensitivity analysis was performed to investigate the above associations after excluding the first year of observation. We also performed a subanalysis of the risk of any stroke, ischaemic stroke and haemorrhagic stroke with PTSD stratified by age groups: young adults ( $<40$ years), middle-aged adults ( $40-59$ years) and older adults (60 years and over). A two-tailed $P$-value of $<0.05$ was considered statistically significant. All data processing and statistical analyses were performed with SPSS version 17 and SAS version 9.1 for Windows.

\section{Results}

In all, 5217 individuals with PTSD and 20868 age- and gendermatched controls were enrolled in our study (Table 1), with an average age of 36.65 years (s.d.=12.76) and a female predominance $(79.1 \%)$. Patients with PTSD lived in less urbanised regions $(P<0.001)$ and had a lower income-related insured amount $(P<0.001)$. The PTSD cohort exhibited an increased incidence of any stroke (3.82 v. 1.00 per 1000 person-years, $P<0.001)$, ischaemic stroke (2.07 v. 0.53 per 1000 person-years,
$P<0.001)$, and haemorrhagic stroke $(0.48$ v. 0.20 per 1000 person-years, $P<0.001)$ and an earlier age at onset of any stroke (55.90 years, s.d. $=15.86$, v. 63.03 years, s.d. $=15.19, P=0.001$ ) and ischaemic stroke (57.53 years, s.d. $=15.59$, v. 63.56 years, s.d. $=12.79, P=0.021)$ compared with the control cohort (Table 2). Stratified by age group, young, middle-aged and older adults all had a significantly higher rates of incidence of stroke than the control group: in young adults the incidence of any stroke was 1.21 v. 0.19 per 1000 person-years $(P<0.001)$, ischaemic stroke was 0.55 v. 0.05 per 1000 person-years $(P<0.001)$ and haemorrhagic stroke was 0.38 v. 0.12 per 1000 person-years $(P<0.001)$ compared with the control group; middle-aged adults had a higher incidence of any stroke (5.57 v. 1.24 per 1000 person-years, $P<0.001)$, ischaemic stroke (3.03 v. 0.83 per 1000 person-years, $P<0.001$ ) and haemorrhagic stroke $(0.53$ v. 0.22 per 1000 person-years, $P<0.001)$ than the control group; older adults also had a higher incidence of any stroke (25.97 v. 9.15 per 1000 person-years, $P<0.001)$, ischaemic stroke ( 14.60 v. 4.30 per 1000 person-years, $P<0.001)$ and haemorrhagic stroke (1.25 v. 1.10 per 1000 personyears, $P<0.001)$ than the control group. Furthermore, compared with the control group, patients with PTSD had significantly higher prevalence rates at enrolment and during the whole follow-up period of major depression, hypertension, dyslipidaemia, diabetes mellitus, ischaemic heart disease, arrhythmia and head injury; for renal disease the difference was significant only at follow-up. Prevalence rates and probabilities are summarised in Table 3.

The Cox regression model showed that PTSD increased the risk of developing any stroke $(\mathrm{HR}=3.37,95 \%$ CI $2.44-4.67)$ and ischaemic stroke ( $\mathrm{HR}=3.47,95 \%$ CI 2.23-5.39) after adjusting for demographic data and medical comorbidities (at enrolment and during the whole follow-up) (Tables 4 and 5). The crude hazard ratio of subsequent haemorrhagic stroke among patients with PTSD was significant $(\mathrm{HR}=2.34,95 \% \mathrm{CI}$ 1.21-4.52), but the significance disappeared after adjusting for demographic data and medical comorbidities ( $\mathrm{HR}=1.35,95 \%$ CI 0.54-3.37). Furthermore, the comorbidities of major depression $(\mathrm{HR}=2.22,95 \% \mathrm{CI} 1.22-4.06)$, hypertension $(\mathrm{HR}=2.04,95 \%$ CI 1.35-3.09) and dyslipidaemia ( $\mathrm{HR}=1.58,95 \%$ CI $1.04-2.41)$ were associated with an increased risk of developing all stroke (Table 4 ); the comorbidities of major depression ( $\mathrm{HR}=2.91,95 \% \mathrm{CI}$ 1.33-6.38) and hypertension ( $\mathrm{HR}=2.96,95 \%$ CI 1.73-5.05) were

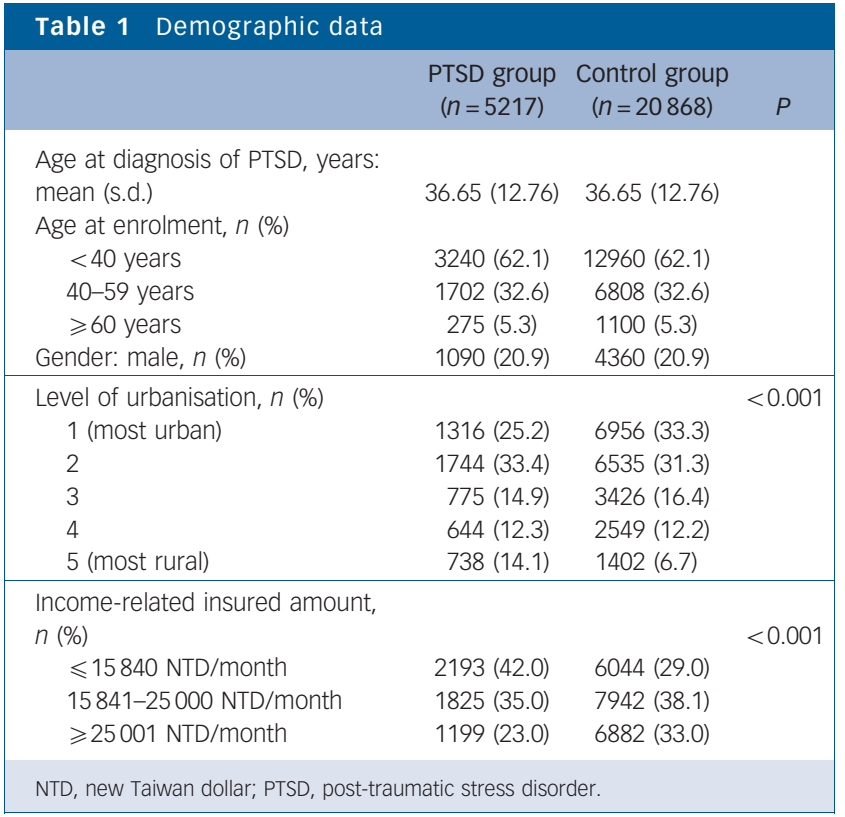


Table 2 Incidence of stroke in the two study groups

PTSD group control group

$(n=5217) \quad(n=20868)$

\section{All stroke \\ Incidence}

$n$

Rate/1000 person-years
Age at diagnosis of stroke, years:

mean (s.d.)

Time between PTSD/enrolment and

diagnosis of stroke, years: mean

(s.d.)

Time between PTSD/enrolment

and diagnosis of stroke, $n(\%)$

$<1$ year

$\geqslant 1$ year

Ischaemic stroke

Incidence

$n$

Rate/1000 person-years

Age at diagnosis of stroke, years:

mean (s.d.)

Time between PTSD/enrolment and

diagnosis of stroke, years: mean

(s.d.)

Time between PTSD/enrolment and

diagnosis of stroke, $n$ (\%)

$\begin{array}{lrr}<1 \text { year } & 14(23) & 1(2) \\ \geqslant 1 \text { year } & 46(77) & 61(98)\end{array}$

Haemorrhagic stroke

Incidence

$n$

Rate/1000 person-years

Age at diagnosis of stroke, years:

mean (s.d.)

Time between PTSD/enrolment and

diagnosis of stroke, years: mean

(s.d.)

Time between PTSD/enrolment and

diagnosis of stroke, $n$ (\%)

$<1$ year

$\geqslant 1$ year

4 (29)

$10(71)$

$0(0)$

24 (100)

PTSD, post-traumatic stress disorder. associated with an increased risk of developing ischaemic stroke; and the comorbidities of hypertension ( $\mathrm{HR}=4.36,95 \% \mathrm{CI}$ 1.73-11.01) and head injury $(\mathrm{HR}=12.69$, 95\% CI 4.99-30.26) were associated with an increased risk of developing haemorrhagic stroke (Table 5). Sensitivity analysis after excluding the first year of observation had consistent findings (Table 6): PTSD increased the risk of developing any stroke $(\mathrm{HR}=3.02,95 \% \mathrm{CI} 2.13-4.28)$ and ischaemic stroke $(\mathrm{HR}=2.89,95 \%$ CI 1.79-4.66). Subanalysis stratified by age further showed consistent findings among the three age groups (Tables 4 and 5). The results showed the risk of stroke following PTSD decreased with age: among young adults the PTSD-related hazard ratios of developing any stroke and ischaemic stroke were 5.36 (95\% CI 2.44-11.77) and 11.53 (95\% CI 3.39-39.24) respectively; among middle-aged adults these risks reduced to 3.82 (95\% CI 2.31-6.32) and 3.56 (95\% CI 1.88-6.72). Older adults had the lowest risk (but still significantly higher) of developing any stroke $(\mathrm{HR}=2.47,95 \%$ CI 1.44-4.23) and ischaemic stroke $(\mathrm{HR}=2.31,95 \%$ CI 1.08-4.92) compared with the control group.

\section{Discussion}

Our results supported the study hypothesis that individuals with PTSD, especially young adults, have an increased risk of developing any stroke and ischaemic stroke in later life. Furthermore, individuals with PTSD developed any stroke and ischaemic stroke at an earlier age than the control group. Previous evidence has reported the association between PTSD and cardiovascular and cerebrovascular risk factors, including hypertension, dyslipidaemia, diabetes mellitus, arrhythmia and smoking..$^{5-9,21-24}$ After analysing the cardiovascular disease risks of 303223 US army veterans after their military service in Iraq and Afghanistan, Cohen et al concluded that both men and women with PTSD had significantly greater rates of tobacco use (men, $\mathrm{OR}=3.63,95 \%$ CI $3.54-3.71$; women, $\mathrm{OR}=3.58,95 \% \mathrm{CI} 3.30-3.88)$, hypertension $(\mathrm{OR}=2.88$, 95\% CI 2.79-2.97; OR $=2.99,95 \%$ CI 2.67-3.33), dyslipidaemia $(\mathrm{OR}=2.70,95 \%$ CI 2.63-2.78; $\mathrm{OR}=2.68,95 \%$ CI 2.44-2.95), obesity $(\mathrm{OR}=2.35,95 \%$ CI $2.27-2.43$; OR $=3.01,95 \%$ CI $2.76-$ 3.28) and diabetes mellitus ( $\mathrm{OR}=2.57,95 \%$ CI 2.37-2.78; $\mathrm{OR}=2.86,95 \%$ CI 2.21-3.71) than those without mental health diagnoses. ${ }^{7}$ Boscarino \& Chang further analysed resting 12-lead electrocardiographic results and cardiovascular comorbidities among 4462 men who were military veterans and found that PTSD was associated with atrioventricular conduction defects $(\mathrm{OR}=2.81,95 \%$ CI 1.03-7.66) and myocardial infarctions $(\mathrm{OR}=4.44,95 \%$ CI $1.20-16.43) .{ }^{22}$ Compatible with these epidemiological findings, our results also demonstrated that individuals with PTSD had a greater prevalence of hypertension, dyslipidaemia, arrhythmia, diabetes mellitus and ischaemic heart disease than those without the disorder. The issues of metabolic syndrome and related complications among individuals with PTSD have gained both public health and clinical attention in recent decades, but in contrast to the well-investigated relationship between PTSD and cardiovascular disease, the relationship between PTSD and stroke is still unclear. ${ }^{10-13}$ As mentioned in the introduction, the cross-sectional study by Spitzer et al and the longitudinal follow-up study by Page \& Brass had conflicting results. ${ }^{12,13}$ The former suggested a significant association between PTSD and stroke, but the latter failed to replicate this finding. In our study, after adjusting for demographic data, cardiovascular comorbidities and other medical diseases, we found a significant association between PTSD and any stroke as well as ischaemic stroke. This finding remained robust and consistent even after excluding the first year of observation. This increased risk of 

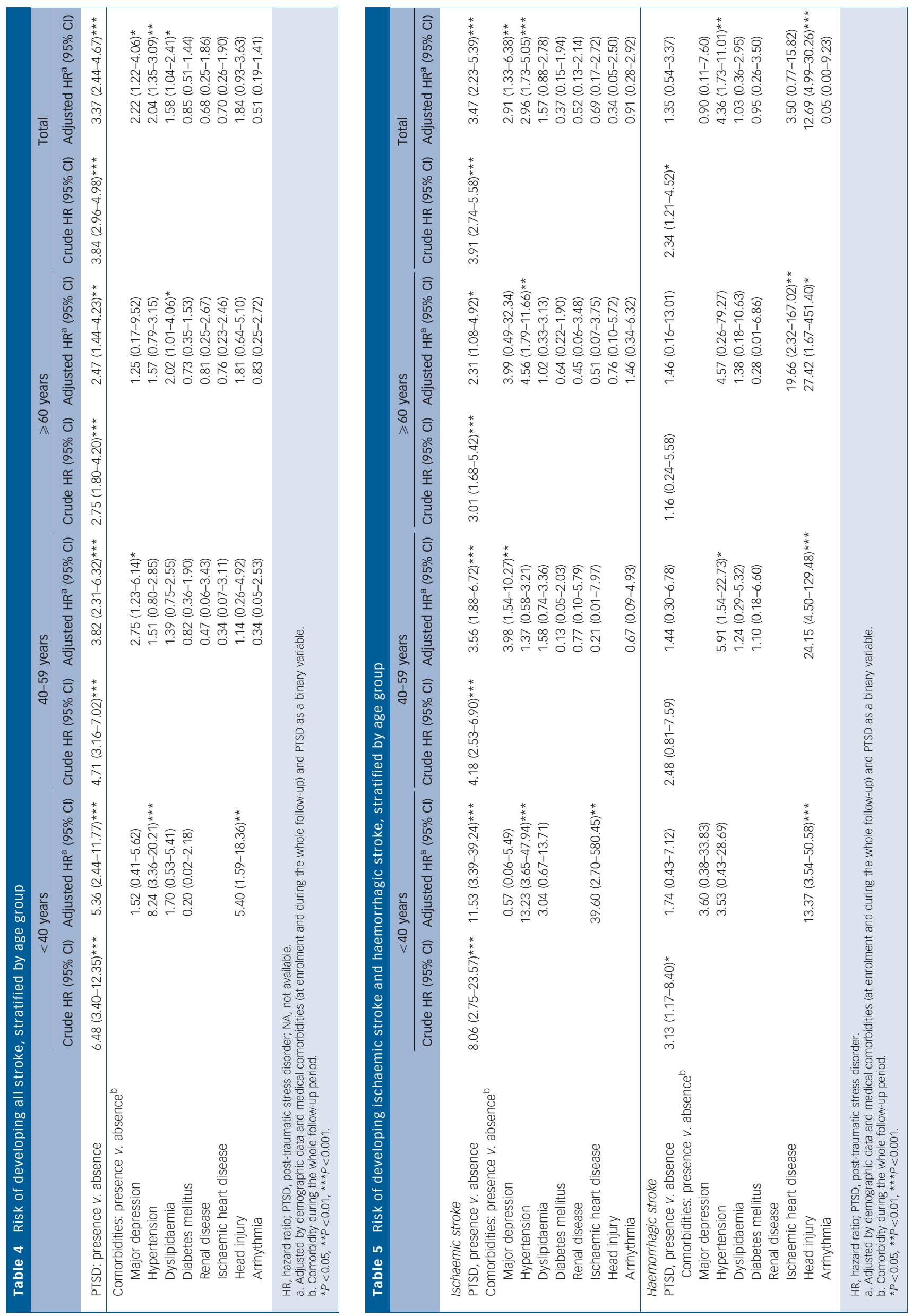
Table 6 Sensitivity test for the risk of developing all stroke, ischaemic stroke and haemorrhagic stroke in the two study groups

\begin{tabular}{|c|c|c|c|c|}
\hline & \multicolumn{2}{|c|}{ Total } & \multicolumn{2}{|c|}{$\geqslant 1$ year } \\
\hline & Crude HR $(95 \% \mathrm{Cl})$ & Adjusted $\mathrm{HR}^{\mathrm{a}}(95 \% \mathrm{Cl})$ & Crude HR $(95 \% \mathrm{Cl})$ & Adjusted $\mathrm{HR}^{\mathrm{a}}(95 \% \mathrm{Cl})$ \\
\hline \multicolumn{5}{|l|}{ All stroke } \\
\hline \multicolumn{5}{|l|}{ PTSD } \\
\hline Absence & 1 & 1 & 1 & 1 \\
\hline Presence & $3.84(2.96-4.98)^{* * *}$ & $3.37(2.44-4.67)^{* * *}$ & $2.95(2.23-3.92)^{* * *}$ & $3.02(2.13-4.28)^{* * *}$ \\
\hline \multicolumn{5}{|c|}{ Ischaemic stroke } \\
\hline \multicolumn{5}{|c|}{ PTSD } \\
\hline Absence & 1 & 1 & 1 & 1 \\
\hline Presence & $3.91(2.74-5.58)^{\star \star \star *}$ & $3.47(2.23-5.39)^{\star \star \star}$ & $3.05(2.08-4.48)^{\star \star \star *}$ & $2.89(1.79-4.66)^{\star \star \star *}$ \\
\hline \multicolumn{5}{|c|}{ Haemorrhagic stroke } \\
\hline \multicolumn{5}{|c|}{ PTSD } \\
\hline Absence & 1 & 1 & 1 & 1 \\
\hline Presence & $2.34(1.21-4.52)^{*}$ & $1.35(0.54-3.37)$ & $1.67(0.80-3.49)$ & $1.10(0.40-2.98)$ \\
\hline
\end{tabular}

developing any stroke and ischaemic stroke was especially observed among young adults with PTSD.

\section{Mechanism of increased risk}

Several hypotheses may explain this association between PTSD and stroke, including dysregulation of the hypothalamic-pituitaryadrenal (HPA) axis, sympathetic adrenal medullary dysfunction, neurochemical dysregulation and endothelial dysfunction. ${ }^{6,21,24,25}$ Research examining the neurophysiology of PTSD has indicated that a crucial feature of the disorder is the altered regulation of stress hormone release by both the HPA axis and the sympathetic adrenal medullary system, including corticotropin-releasing hormone, corticosteroid and catecholamine. ${ }^{6,21,24,25}$ Individuals with PTSD exhibited a paradoxical phenomenon of low levels of plasma and urinary cortisol and elevated levels of corticotropinreleasing hormone. This phenomenon owing to the increased sensitivity of the HPA axis to negative feedback may result in a dysregulated immune state, dysregulation of lipid and glucose metabolism, and altered brain function. ${ }^{24,26}$ Previous studies demonstrated that higher levels of proinflammatory cytokines, including tumour necrosis factor $\alpha$ and interleukins IL- 6 and IL-1 $\beta$, appeared in individuals with PTSD compared with the controls, and were positively correlated with duration of PTSD symptoms. ${ }^{27,28}$ The PTSD-related dysregulated secretions of proinflammatory cytokines had more far-reaching effects, such as accelerated injury to the vascular endothelium and ultimately the development of cardiovascular disease. ${ }^{24,27,28}$ Another important factor related to the cardiovascular and cerebrovascular risks was the perturbation in autonomic nervous activity, namely enhanced sympathetic and diminished parasympathetic functions. $5,6,23,24$ Yehuda et al found that individuals with PTSD had significantly higher levels of dopamine, noradrenaline (norepinephrine) and adrenaline (epinephrine) in their $24 \mathrm{~h}$ urine analysis than the controls, and suggested that levels of dopamine and noradrenaline were significantly correlated with severity of PTSD symptoms. ${ }^{29}$ Dikanoviæ et al further reported an association between elevated levels of $24 \mathrm{~h}$ vanillylmandelic acid (an adrenaline and noradrenaline metabolite) and increased values of mean blood flow velocity and vasospasm in the circle of Willis vasculature among individuals with PTSD. ${ }^{30}$ The imbalance in autonomic activity resulting in elevated catecholamine levels, increased resting heart rate, higher blood pressure and reduced cardiac vagal tone would increase the risk of cardiovascular and cerebrovascular disease. ${ }^{24}$ Finally, individuals with PTSD may be more susceptible to vascular endothelial injuries. 5,6,23,24 Ahmadi et al calculated the coronary artery calcium score (a measure of atherosclerotic risk) of 637 people in an elderly veterans sample, and found that the scores were positively correlated with PTSD symptoms and that PTSD was an independent predictor of the presence of atherosclerotic coronary artery disease. ${ }^{31}$ Von Kanel et al further showed that PTSD symptoms were correlated significantly and positively with the markers of endothelial dysfunction (i.e. soluble tissue factor and von Willebrand factor). ${ }^{32}$ Combining the above evidence, it can be seen that PTSD may increase the risk of cerebrovascular diseases through disrupted regulation of the HPA axis and autonomic nervous system, increased secretion of proinflammatory cytokines, endothelial dysfunction and related coagulopathy, and insulin resistance. ${ }^{24}$ Our results indicated that PTSD was associated with an increased risk of developing any stroke and ischaemic stroke after adjusting for demographic data and stroke-related medical comorbidities. Further studies would be required to validate this finding and clarify the pathophysiology underlying PTSD and stroke.

No previous study had investigated the relationship between PTSD and subsequent haemorrhagic stroke, and the risk of such stroke among patients with PTSD was still unknown. In our study, the crude hazard ratio of haemorrhagic stroke among patients with PTSD was statistically significant, but this significance disappeared after adjusting for medical comorbidities. It may indicate that the association between PTSD and haemorrhagic stroke was indirectly due to other medical comorbidities, such as hypertension and head injury. However, further studies would be required to elucidate this possible association between PTSD and haemorrhagic stroke.

Finally, we found that young adults with PTSD had even a higher hazard ratio of developing any stroke and ischaemic stroke than the elderly group with PTSD. Previous studies also suggested that the incidence of stroke among young adults was much lower than the incidence among older people. It may explain the reason why the adjusted HR decreased with age groups in our sample. Further studies may be required to validate the association between young patients with PTSD and subsequent stroke, and investigate the possible underlying mechanisms.

\section{Study limitations}

Some limitations should be addressed here. First, the incidence of stroke may have been underestimated because only individuals who sought medical help were enrolled. However, the individuals enrolled in our study had board-certified physician diagnoses, yielding better diagnostic validity. Second, the NHIRD did not 
provide information about the severity of PTSD symptoms so we could not investigate the association between PTSD severity and the risk of stroke; further clinical studies would be required to clarify this association. Third, the NHIRD did not provide information about the previous history of PTSD. False negatives in the control group may exist, although we excluded everyone who had been given a diagnosis of PTSD at any time from the control group. Fourth, the NHIRD did not provide information such as smoking history, details of traumatic events, family history, personal lifestyle and environmental factors. Without this information, we were unable to examine their influence.

\section{Study implications}

Our results supported the idea that PTSD is associated with an elevated risk of developing any stroke and ischaemic stroke in later life after adjusting for demographic data and medical comorbidities. Further studies are required to investigate the underlying pathophysiology relating PTSD and stroke.

\footnotetext{
Mu-Hong Chen, MD, Department of Psychiatry, Taipei Veterans General Hospital, Taipei, Department of Psychiatry, College of Medicine, National Yang-Ming University, Taipei; Tai-Long Pan, PhD, School of Traditional Chinese Medicine, Chang Gung Taipel; Tai-Long Pan, PhD, School of Traditional Chinese Medicine, Chang Gung
University, Taoyuan, and Research Center for Industry of Human Ecology, Chang Gung University of Science and Technology, Taoyuan, and Liver Research Center, Division of Hepatology, Department of Gastroenterology and Hepatology, Chang Gung Memorial Hospital, Taoyuan; Cheng-Ta Li, MD, PhD, Wei-Chen Lin, MD, Ying-Sheue Chen, MD, Ying-Chiao Lee, MD, Shih-Jen Tsai, MD, Ju-Wei Hsu, MD, Kai-Lin Huang, MD, Chia-Fen Tsai, MD, Department of Psychiatry, Taipei Veterans General Hospital, Taipei, Department of Psychiatry, College of Medicine, National Yener-Ming University, Taipei; Wen-Han Chang, MSc, Department of Psychiatry,
Yang-Ming Taipei Veterans General Hospital, Taipei; Tzeng-Ji Chen, MD, PhD, Department of Family Medicine, Taipei Veterans General Hospital, Institute of Hospital and Health Care Administration, National Yang-Ming University, Taipei; Tung-Ping Su, MD, Ya-Mei Bai, MD, PhD, Department of Psychiatry, Taipei Veterans General Hospital, Taipei, Department of Psychiatry, College of Medicine, National Yang-Ming University, Taipei, Taiwan

Correspondence: Dr Ya-Mei Bai, Department of Psychiatry, Taipei Veterans General Hospital, No.201, Sec. 2, Shipai Road, Beitou District, Taipei 112, Taiwan. Email: ymbi@mail2000.com.tw
}

First received 17 Dec 2013, final revision 30 Jun 2014, accepted 19 Sep 2014

\section{Funding}

The study was supported by grant from the Taipei Veterans General Hospital (V103E10-001)

\section{Acknowledgements}

We thank Mr I-Fan Hu for his friendship and support.

\section{References}

1 Stein DJ, Seedat S, Iversen A, Wessely S. Post-traumatic stress disorder medicine and politics. Lancet 2007; 369: 139-44.

2 Yehuda R. Post-traumatic stress disorder. N Engl J Med 2002; 346: 108-14

3 Kessler RC, Sonnega A, Bromet E, Hughes M, Nelson CB. Posttraumatic stress disorder in the National Comorbidity Survey. Arch Gen Psychiatry 1995; 52: 1048-60

4 Chou FH, Wu HC, Chou P, Su CY, Tsai KY, Chao SS, et al. Epidemiologic psychiatric studies on post-disaster impact among Chi-Chi earthquake survivors in Yu-Chi, Taiwan. Psychiatry Clin Neurosci 2007; 61: 370-8.

5 Bartoli F, Carra G, Crocamo C, Carretta D, Clerici M. Metabolic syndrome in people suffering from posttraumatic stress disorder: a systematic review and meta-analysis. Metab Syndr Relat Disord 2013; 11: 301-8.

6 Bedi US, Arora R. Cardiovascular manifestations of posttraumatic stress disorder. J Natl Med Assoc 2007; 99: 642-9.

7 Cohen BE, Marmar C, Ren L, Bertenthal D, Seal KH. Association of cardiovascular risk factors with mental health diagnoses in Iraq and Afghanistan war veterans using VA health care. JAMA 2009; 302: 489-92.

8 Kibler JL, Joshi K, Ma M. Hypertension in relation to posttraumatic stress disorder and depression in the US National Comorbidity Survey. Behav Med 2009; 34: 125-32.
9 Lukaschek K, Baumert J, Kruse J, Emeny RT, Lacruz ME, Huth C, et al. Relationship between posttraumatic stress disorder and type 2 diabetes in a population-based cross-sectional study with 2970 participants. J Psychosom Res 2013; 74: 340-5.

10 Brass LM, Page WF. Stroke in former prisoners of war. J Stroke Cerebrovasc Dis 1996; 6: 72-8.

11 Coughlin SS. Post-traumatic stress disorder and cardiovascular disease. Open Cardiovasc Med 2011; 5: 164-70.

12 Page WF, Brass LM. Long-term heart disease and stroke mortality among former American prisoners of war of World War II and the Korean Conflict: results of a 50-year follow-up. Mil Med 2001; 166: 803-8.

13 Spitzer C, Barnow S, Volzke H, John U, Freyberger HJ, Grabe HJ. Trauma, posttraumatic stress disorder, and physical illness: findings from the general population. Psychosom Med 2009; 71: 1012-7.

14 World Health Organization. International Classification of Diseases, Ninth Revision, Clinical Modification (ICD-9-CM). WHO, 2009.

15 Bai YM, Su TP, Chen MH, Chen TJ, Chang WH. Risk of developing diabetes mellitus and hyperlipidemia among patients with bipolar disorder, major depressive disorder, and schizophrenia: a 10-year nationwide populationbased prospective cohort study. J Affect Disord 2013; 150: 57-62.

16 Chen MH, Su TP, Chen YS, Hsu JW, Huang KL, Chang WH, et al. Attention deficit hyperactivity disorder, tic disorder, and allergy: is there a link? A nationwide population-based study. J Child Psychol Psychiatry 2013; 54: 545-51.

17 Chen MH, Su TP, Chen YS, Hsu JW, Huang KL, Chang WH, et al. Asthma and attention-deficit/hyperactivity disorder: a nationwide population-based prospective cohort study. J Child Psychol Psychiatry 2013; 54: 1208-14.

18 Shen CC, Tsai SJ, Perng CL, Kuo BI, Yang AC. Risk of Parkinson disease after depression: a nationwide population-based study. Neurology 2013; 81: 1538-44.

19 Wu CY, Chen YJ, Ho HJ, Hsu YC, Kuo KN, Wu MS, et al. Association between nucleoside analogues and risk of hepatitis $B$ virus-related hepatocellular carcinoma recurrence following liver resection. JAMA 2012; 308: 1906-14.

20 Liu CY, Hung YT, Chuang YL, Chen YJ, Weng WS, Liu JS. Incorporating development stratification of Taiwan townships into sampling design of large scale health interview survey. J Health Management (Chin) 2006; 4: 1-22.

21 Boscarino JA. Post-traumatic stress disorder and cardiovascular disease link: time to identify specific pathways and interventions. Am J Cardiol 2011; 108: 1052-3.

22 Boscarino JA, Chang J. Electrocardiogram abnormalities among men with stress-related psychiatric disorders: implications for coronary heart disease and clinical research. Ann Behav Med 1999; 21: 227-34.

23 Levine AB, Levine LM, Levine TB. Posttraumatic stress disorder and cardiometabolic disease. Cardiology 2013; 127: 1-19.

24 Wentworth BA, Stein MB, Redwine LS, Xue Y, Taub PR, Clopton P, et al. Post-traumatic stress disorder: a fast track to premature cardiovascular disease? Cardiol Rev 2013; 21: 16-22.

25 Sawchuk CN, Roy-Byrne P, Goldberg J, Manson S, Noonan C, Beals J, et al. The relationship between post-traumatic stress disorder, depression and cardiovascular disease in an American Indian tribe. Psychol Med 2005; 35: 1785-94.

26 Bremner JD, Vythilingam M, Anderson G, Vermetten $\mathrm{E}$, McGlashan $\mathrm{T}$, Heninger $\mathrm{G}$, et al. Assessment of the hypothalamic-pituitary-adrenal axis over a 24-hour diurnal period and in response to neuroendocrine challenges in women with and without childhood sexual abuse and posttraumatic stress disorder. Biol Psychiatry 2003; 54: 710-8.

27 Spivak B, Shohat B, Mester R, Avraham S, Gil-Ad I, Bleich A, et al. Elevated levels of serum interleukin-1 beta in combat-related posttraumatic stress disorder. Biol Psychiatry 1997; 42: 345-8.

28 Tucker P, Ruwe WD, Masters B, Parker DE, Hossain A, Trautman RP, et al. Neuroimmune and cortisol changes in selective serotonin reuptake inhibitor and placebo treatment of chronic posttraumatic stress disorder. Biol Psychiatry 2004; 56: 121-8.

29 Yehuda R, Southwick S, Giller EL, Ma X, Mason JW. Urinary catecholamine excretion and severity of PTSD symptoms in Vietnam combat veterans. J Nerv Mental Dis 1992; 180: 321-5.

30 Dikanovic M, Demarin V, Kadojic D, Kadojic M, Trkanjec Z, Titlic M, et al. Effect of elevated catecholamine levels on cerebral hemodynamics in patients with chronic post-traumatic stress disorder. Coll Antropol 2011; 35 471-5.

31 Ahmadi N, Hajsadeghi F, Mirshkarlo HB, Budoff M, Yehuda R, Ebrahimi R. Post-traumatic stress disorder, coronary atherosclerosis, and mortality. Am J Cardiol 2011; 108: 29-33.

32 Von Kanel R, Hepp U, Traber R, Kraemer B, Mica L, Keel M, et al. Measures of endothelial dysfunction in plasma of patients with posttraumatic stress disorder. Psychiatry Res 2008; 158: 363-73. 